\title{
Toward the understanding of autophagy regulation and its interplay with cell death pathways
}

\author{
GM Fimia ${ }^{1}$ and M Piacentini ${ }^{*, 1,2}$ \\ Cell Death and Differentiation (2009) 16, 933-934; doi:10.1038/cdd.2009.47
}

The growing importance of autophagy is clearly shown by the exponentially increasing number of publications appearing on a daily basis in PubMed. Although initially autophagy was classified as a form of cell death, it is now generally accepted that this is not the case and that it mainly promotes cell health and survival and, only under peculiar conditions, can it result in cellular death. In the first issue of 2009, Cell Death and Differentiation has already published nine reviews on the topic of autophagy in aging, disease and death. ${ }^{1-9}$ These are now followed by another six papers covering more specific aspects of the complex regulation of this process and its crosstalk with apoptosis and other essential cellular functions, such as energy production, vesicular traffic and cellular immunity. ${ }^{10-15}$

Three reviews focus on the molecular mechanisms regulating the induction, formation and maturation of the autophagosome vesicles. Longatti and Tooze $^{10}$ give a comprehensive view of the proteins regulating the initial events during autophagosome formation in higher eukaryotes. The activation of ULK kinases is an early regulatory step essential for the induction of many downstream autophagic events. However, a full comprehension of function of these kinases still requires a complete identification of their autophagy-specific protein substrates. After the induction of the ULK kinases, the Beclin 1-Class III PI3-Kinase complex is activated to produce PI3-P-enriched membrane domains, which are probably acting as platforms able to recruit the downstream factors, (Atg5-12-16 complex, LC3 and ATG9) which are required for autophagosomes assembly. In this regard, the authors propose four alternative models to explain how the autophagic vesicles are formed (de novo synthesis, vesicular transport, cisternal assembly, membrane remodeling). However, there is no definitive evidence favouring one of these models, and the molecular mechanisms regulating autophagosome biogenesis remain to be elucidated, despite the extensive research on this subject.

A further insight into the formation of the autophagosome is presented by Takahashi et al., ${ }^{11}$ who focus their attention on the role of Bif-1 in the autophagic process. Bif-1, is a BAR domain-containing protein belonging to the endophilin family, which, by binding membranes, causes their curvature. By interacting with UVRAG, Bif-1 is recruited on the Beclin
1-Class III PI3K complex, indicating that, beside its role in lipid phosphorylation, this complex could also engage factors required for autophagosome expansion.

Recent advances in the late steps of the autophagic process, from the expansion of the autophagosomes to their fusion with lysosomes, are described in the review by Noda et al. ${ }^{12}$ In particular, the authors focus on the role of LC3, the mammalian homolog of yeast ATG8. On the basis of recent experimental data, the authors propose that the main function of LC3 is related to the closure of the autophagosomes by a mechanism named 'reverse fusion'. In this model, the authors suggest a novel hypothesis on how the leading edges of the isolation membrane can converge and fuse. The final delivery of mature autophagosomes to the lysosomes is achieved by a mechanism shared with other vesicle trafficking pathways. In this event, a key role is played by UVRAG, which, in addition to its function in the Beclin 1 complex, is also a component of the Class $\mathrm{C}$ Vps/HOPS proteins involved in the fusion mechanism.

Three other papers in this review set describe interplay between autophagy and apoptosis in determining cell fate. ${ }^{13-15}$ The crosstalk between these two events is discussed in the review by Eisenberg-Lerner et al. ${ }^{13}$ The original concept that inhibition of apoptosis results in autophagic/necrotic cell death is now being extended and it is becoming clear that apoptosis and autophagy can act as partners to induce cell death in a coordinated or cooperative manner. In fact, autophagy can facilitate cell death by enabling cellular events that occur during apoptosis. It has recently been shown that autophagy is upstream of apoptosis in ER stress-induced cancer cell death, acting as an antagonist; ${ }^{16}$ in fact, the ablation of Ambra1 and ATG5, which inhibit autophagy, reduces apoptosis in glioma cells treated with cannabinoids. ${ }^{16}$ By contrast, in some settings autophagy acts by promoting cell survival, as an antagonist of apoptosis. For example, it has been shown that autophagy can play a positive role through the degradation of protein aggregates and misfolded proteins, thus limiting the ER-stress response and subsequent apoptosis. ${ }^{5}$ There is a growing number of proteins that have been shown to play a regulatory role in both events. Particularly interesting is the dual regulation of autophagy carried out by p53, which depends on its intracellular localization; in fact, nuclear resident p53 favors

\footnotetext{
${ }^{1}$ National Institute for Infectious Diseases, Via Portuense 292, Rome 00149, Italy and ²Department of Biology, University of Rome 'Tor Vergata', Via della Ricerca Scientifica 1, Rome 00133, Italy

*Corresponding author: M Piacentini, Department of Biology, University of Rome Tor Vergata, Via della Ricerva Scientifica 1, Rome 133, Italy. Tel: + 390672594234 ; Fax: + 39062023 500; E-mail: mauro.piacentini@uniroma2.it
} 
induction of autophagy, whereas cytoplasmic accumulation represses basal pro-survival autophagy. ${ }^{6}$ The $\mathrm{Bcl}-2$ family members bind and inhibit Beclin 1; interestingly, this interaction involves a $\mathrm{BH} 3$ domain within Beclin-1. It is becoming evident that the protein-protein interaction regulated by $\mathrm{BH} 3$ domains seems to be essential not only in the regulation of apoptosis but also in that of autophagy. ${ }^{14}$ In line with this assumption, Zhang and $\mathrm{Ney}^{14}$ discuss the role in cell death and autophagy of the BH3-only proteins BNIP3 and BNIP3like (BNIP3L), also known as NIX. The molecular mechanisms by which BNIP3 and NIX induce both cell death and autophagy are not well understood. BNIP and NIX are weak inducers of cell death and their activity is primarily determined by the transmembrane domain and involves the opening of the mitochondrial permeability transition pore. Unlike typical BH3-only proteins, which cause apoptotic cell death, BNIP3 and NIX induce cell death by necrosis. These proteins are important in the regulation of the mitochondrial homeostasis and mitophagy. In fact, analysis of NIX-deficient mice has shown a defect in erythroid development that is not directly related to the regulation of cell death, but to an impairment of mitochondrial clearance during erythrocyte differentiation. Thus, BNIP3 and NIX exhibit a dual nature; they induce cell death by necrosis, and they also participate in the induction of autophagy, very likely affecting the binding of Beclin 1 to the Bcl-2 family members. Another key effector of both processes is the DAPk, which, catalyzing the phosphorylation of multiple substrates plays a complex role in apoptosis regulation. Recently, Beclin 1 has been shown to be among the substrates of this kinase and its phosphorylation reduces the binding to the Bcl-2 family members, thus suggesting a possible mechanism by which DAPk may also induce autophagy. $^{13}$

An interesting clue about the importance of autophagy for cell death induction in mammals derives from knockout studies in mice ablated of essential autophagy genes, such as ambra 1 , beclin 1 and atg5. ${ }^{17-19}$ These animals die during development showing an increased numbers of apoptotic cells in their tissues, thus, indicating the existence of a crosstalk between these two essential cell pathways already during embryogenesis.

Another key scenario in which the interplay between autophagy and apoptosis perform an essential role is the immune system homeostasis. The importance of autophagy in both innate and adaptive immunity has been recently proposed. ${ }^{8}$ In fact, autophagy controls viral infections, the replication of intracellular bacteria and parasites and it also contributes to delivering cytoplasmic antigens for MHC class II presentation during the adaptive immune system response. Thus, the immune system utilizes autophagy to eliminate intracellular pathogens and regulates adaptive immunity and apoptosis to eliminate irremediably compromised infected cells by cell-mediated cytotoxicity and for many other essential protective and homeostatic actions. In many settings, the interplay between survival and death-promoting signals is regulated by the assembly of pathway-specific protein complexes competing with each other, until one eventually dominates resulting in apoptosis or autophagy. ${ }^{20}$ In the review by Delgato and Deretic, ${ }^{15}$ the relationships between autophagy and pattern recognition receptors (PRRs), particularly the Toll-like receptors (TLR), are carefully discussed. This topic is of particular importance as PRRs responses, together with their downstream signaling and effector outputs are needed for the clearance of pathogens. However, they have to be tightly controlled to avoid an excessive response that could result in the induction of apoptosis with severe consequence for the host. An example of the molecular switches between these pathways is represented by the alternative binding of the crucial adaptor kinase, RIP1, to the different effector protein complexes at the crossroad of a cell's decision to survive or die. ${ }^{20}$

Future studies should definitively unravel the molecular mechanisms regulating the complex interplay between autophagy and cell death. We believe that the review papers included in this issue of Cell Death and Differentiation represent an excellent basis to tackle these scientific challenges.

1. Scarlatti F, Granata R, Meijer AJ, Codogno P. Does autophagy have a license to kill mammalian cells? Cell Death Differ 2009; 16: 12-20.

2. Kourtis N, Tavernarakis N. Utophagy and cell death in model organisms. Cell Death Differ 2009; 16: 21-30.

3. Nishida K, Kyoi S, Yamaguchi O, Sadoshima J, Otsu K. The role of autophagy in the heart. Cell Death Differ 2009; 16: 31-38.

4. Perlmutter $\mathrm{DH}$. Autophagic disposal of the aggregation-prone protein that causes liver inflammation and carcinogenesis in alpha-1-antitrypsin deficiency. Cell Death Differ 2009; 16: $39-45$.

5. Sarkar S, Ravikumar B, Floto RA, Rubinsztein DC. Rapamycin and mTOR-independent autophagy inducers ameliorate toxicity of polyglutamine-expanded huntingtin and related proteinopathies. Cell Death Differ 2009; 16: 46-56.

6. Maiuri MC, Tasdemir E, Criollo A, Morselli E, Vicencio JM, Carnuccio R et al. Control of autophagy by oncogenes and tumor suppressor genes. Cell Death Differ 2009; 16: 87-93.

7. Orvedahl A, Levine B. Eating the enemy within: autophagy in infectious diseases. Cell Death Differ 2009; 16: 57-69.

8. Lünemann JD, Münz C. Autophagy in CD4+ T-cell immunity and tolerance. Cell Death Differ 2009; 16: 79-86.

9. Vellai T. Autophagy genes and ageing. Cell Death Differ 2009; 16: 94-1024.

10. Longatti A, Tooze SA. Cell Death Differ 2009; 16: 956-965.

11. Takahashi Y, Meyerkord CL, Wang HG. Bif-1/Endophilin B1: a candidate for crescent driving force in autophagy. Cell Death Differ 2009; 16: 947-955.

12. Noda T, Fujita N, Yoshimori T. Cell Death Differ 2009; 16: 984-990.

13. Eisenberg-Lerner A, Bialik S, Simon HU, Kimchi A. Life and death partners: apoptosis, autophagy and the cross-talk between them. Cell Death Differ 2009; 16: 966-975.

14. Zhang J, Ney PA. Role of BNIP3 and NIX in cell death, autophagy, and mitophagy. Cell Death Differ 2009; 16: 939-946.

15. Delgado MA, Deretic V. Cell Death Differ 2009; 16: 976-983.

16. Salazar $M$ et al. J Clin Invest 2009 , in press.

17. Fimia GM, Stoykova A, Romagnoli A, Giunta L, Di Bartolomeo S, Nardacci R et al. Ambra1 regulates autophagy and development of the nervous system. Nature 2007; 447 1121-1125.

18. Qu X, Zou Z, Sun Q, Luby-Phelps K, Cheng P, Hogan RN et al. Autophagy genedependent clearance of apoptotic cells during embryonic development. Cell 2007; 128: 931-946.

19. Hara T, Nakamura K, Matsui M, Yamamoto A, Nakahara $Y$, Suzuki-Migishima R et al. Suppression of basal autophagy in neural cells causes neurodegenerative disease in mice. Nature 2006; 441: 885-889.

20. Festjens N, Vanden Berghe T, Cornelis S, Vandenabeele P. RIP1, a kinase on the crossroads of a cell's decision to live or die. Cell Death Differ 2007; 14: 400-410. 